\title{
Effects of conditioned medium from LL-37 treated adipose stem cells on human fibroblast migration
}

\author{
EUN-JUNG YANG ${ }^{1}$ and SA-IK BANG ${ }^{2}$ \\ ${ }^{1}$ Department of Plastic Surgery, Cheil General Hospital and Women's Healthcare Center, \\ College of Medicine, Dankook University; ${ }^{2}$ Department of Plastic Surgery, Samsung Medical Center, \\ School of Medicine, Sungkyunkwan University, Seoul 06351, Republic of Korea
}

Received November 24, 2016; Accepted May 19, 2017

DOI: $10.3892 /$ etm.2017.4558

\begin{abstract}
Adipose stem cell-conditioned medium may promote human dermal fibroblast (HDF) proliferation and migration by activating paracrine peptides during the re-epithelization phase of wound healing. Human antimicrobial peptide LL-37 is upregulated in the skin epithelium as part of the normal response to injury. The effects of conditioned medium (CM) from LL-37 treated adipose stem cells (ASCs) on cutaneous wound healing, including the mediation of fibroblast migration, remain to be elucidated, therefore the aim of the present study was to determine how ASCs would react to an LL-37-rich microenvironment and if CM from LL-37 treated ASCs may influence the migration of HDFs. The present study conducted migration assays with HDFs treated with CM from LL-37 treated ASCs. Expression of CXC chemokine receptor 4 (CXCR4), which controls the recruitment of HDFs, was analyzed at the mRNA and protein levels. To further characterize the stimulatory effects of LL-37 on ASCs, the expression of stromal cell-derived factor- $1 \alpha$ (SDF-1 $\alpha$ ), a CXC chemokine, was investigated. CM from LL-37-treated ASCs induced migration of HDFs in a time- and dose-dependent manner, with a maximum difference in migration observed $24 \mathrm{~h}$ following stimulation with LL-37 at a concentration of $10 \mu \mathrm{g} / \mathrm{ml}$. The HDF migration and the expression of CXCR4 in fibroblasts was markedly increased upon treatment with $\mathrm{CM}$ from LL-37-treated ASCs compared with CM from untreated ASCs. SDF-1 $\alpha$ expression was markedly increased in CM from LL-37 treated ASCs. It was additionally observed that SDF-1 $\alpha$ blockade significantly reduced HDF migration. These findings suggest the feasibility of CM from LL-37-treated ASCs as a potential therapeutic for human dermal fibroblast migration.
\end{abstract}

Correspondence to: Professor Sa-Ik Bang, Department of Plastic Surgery, Samsung Medical Center, School of Medicine, Sungkyunkwan University, 81 Irwon-ro, Gangnam, Seoul 06351, Republic of Korea

E-mail: enyang7@gmail.com

Key words: fibroblast migration, ASC, LL-37, conditioned medium

\section{Introduction}

Cutaneous wound healing is a dynamic process involving the migration and interaction of cells in the dermis and epidermis, including fibroblasts and keratinocytes, as well as the release of chemical mediators from inflammatory processes. In the early phase of wound healing, fibroblasts migrate into the affected area and move across the provisional fibrin-based matrix. Since the provisional fibrin-based matrix is relatively devoid of fibroblasts, the processes of migration, proliferation and exracellular mattress production are considered key steps in the regeneration of functional dermis (1). Moreover, many of growth factors and cytokines are involved in the regulation of fibroblast migration $(2,3)$.

Previous studies showed ASCs may contribute to tissue injury repair. ASCs secrete various growth factors that manage damaged neighboring cells (4). Conditioned medium from ASC cultures (ASC-CM) activates dermal fibroblasts and keratinocytes, and can repair the skin through a paracrine mechanism $(1,5)$. LL-37 is a naturally occurring antimicrobial peptide found in wound beds that has promoting effects on immune cells (6-9). Although both ASCs and LL-37 are suggested as wound healing associators, the relationship between ASCs and LL-37 has not been clarified. Furthermore, no information is available about the effect of LL-37 regulated ASCs on the mediation of the fibroblast migration, which in turn may accelerated wound healing process, and related mechanism. We hypothesized that LL-37 pretreatment to ASCs would enhance secretion of active peptides from ASCs participating in wound healing in a paracrine fashion, and that the resulting recruitment of human dermal fibroblasts (HDFs) to the wound microenvironment would support wound healing. To this end, we investigated the ability of CM from LL-37 treated ASCs to influence HDFs migration in vitro by up-regulating CXC chemokine receptor 4 (CXCR4) and SDF-1 $\alpha$ expression.

\section{Materials and methods}

Cell culture. Human dermal fibroblasts (HDFs) from neonatal foreskin were obtained from the American Type Culture Collection (Manassas, VA, USA). Cells were cultured in a humidified atmosphere containing $5 \% \mathrm{CO}_{2}$ in Dulbecco's 
modified Eagle's medium (DMEM) /high glucose (Thermo Fisher Scientific, Pittsburgh, PA, USA) supplemented with $100 \mathrm{U} / \mathrm{ml}$ penicillin, $100 \mu \mathrm{g} / \mathrm{ml}$ streptomycin, and $10 \%$ fetal bovine serum (FBS; Thermo Fisher Scientific) and maintained at $37^{\circ} \mathrm{C}$.

Preparation of adipose stem cell-conditioned media. Subcutaneous adipose tissue was obtained during elective surgeries with patient consent; this procedure was approved by the Samsung Medical Center Institutional Review Board (IRB). To isolate ASCs, adipose tissue was treated with an equal volume of a $0.1 \%$ collagenase type I (Sigma, St. Louis, MO, USA) solution for $60 \mathrm{~min}$ at $37^{\circ} \mathrm{C}$ with intermittent shaking. Floating adipocytes were separated from the stromal vascular fraction by centrifugation at $1,500 \mathrm{rpm}$ for $10 \mathrm{~min}$. The cell pellet was suspended in DMEM/low glucose supplemented with $10 \% \mathrm{FBS}, 100 \mathrm{U} / \mathrm{ml}$ penicillin, and $100 \mu \mathrm{g} / \mathrm{ml}$ streptomycin, and cells were plated in tissue culture dishes. Primary ASCs were cultured for 4-5 days until they reached confluence and, at this point, were defined as passage 0 . ASCs were used between passages 4 and 6 for experiments. In some experiments, cells were pretreated with $0.5 \mu \mathrm{g} / \mathrm{ml}$ neutralizing LL-37 antibody (HyCult Biotechnology, B.V., Uden, The Netherlands), $10 \mu \mathrm{g} / \mathrm{ml}$ neutralizing SDF-1 $\alpha$ antibody (R\&D systems, MN, USA), and/or $100 \mathrm{ng} / \mathrm{ml} \mathrm{PTX}$ (Sigma) for $60 \mathrm{~min}$ before the addition of human LL-37 (Phoenix Pharmaceuticals, Inc., Burlingame, CA, USA). Conditioned media $(\mathrm{CM})$ was collected from ASC cultures after $48 \mathrm{~h}$ and subjected to filtration ( $0.2 \mu$ filter).

Migration assay. For the transwell migration assay, HDFs cells were plated in the upper chamber (70,000 cells/upper chamber) of 6.5-mm diameter, $8-\mu \mathrm{m}$ pore size transwell inserts (Corning Inc., Acton, MA, USA) in 24-well plates. Prior to plating cells, ASCs were treated with hLL-37 $(10 \mathrm{ug} / \mathrm{ml})$ in media containing $0.5 \%$ FBS for $48 \mathrm{~h}$. The CM from LL-37 treated ASCs was then added to the lower chamber of 24-well plates. After $24 \mathrm{~h}$, the transwell inserts were placed in a fresh 24-well plate containing $350 \mu \mathrm{l}$ of $0.05 \%$ crystal violet, incubated for $20 \mathrm{~min}$, removed, washed by flooding with tap water until free dye was no longer visible, and allowed to air dry.

Real time-PCR. Total RNA was extracted using Trizol reagent (Invitrogen Life Technologies, Carlsbad, CA, USA). cDNA was synthesized using $2 \mu \mathrm{g}$ of total RNA and SuperScript II reverse transcriptase (Invitrogen Life Technologies) according to the manufacturer's instructions. For Real-Time PCR, quantitative PCRs (qPCRs) were performed using the 7900 Real-Time PCR System (Applied Biosystems, Foster City, CA, USA) with the HotStart-IT SYBR-Green qPCR Master Mix kit (USB Corporation, Camberley, UK). The cycling profile for Real-Time PCR (50 cycles) was as follows: $95^{\circ} \mathrm{C}$ for $10 \mathrm{~min}$, $95^{\circ} \mathrm{C}$ for $15 \mathrm{sec}$, and $60^{\circ} \mathrm{C}$ for $60 \mathrm{sec}$. The primers used were as follows: GAPDH forward, 5'-ATCACCATCTTCCAGGAG CGA-3'; reverse, 5'-TTCTCCATGGTGGTGAAGACG-3'; for CXCR4 forward, 5'-TCCGTGAAGAAAATGCTAAT-3'; reverse, 5'-GTAGATGACATGGACTGCCT-3'; SDF-1 $\alpha$ forward, 5'-CTAACTCTCTCCCCGACTCCG-3'; reverse, 5'-AAGCAGGGGGACCATTACAC-3'. The comparative quantification cycle $(\mathrm{Cq})$ method, i.e., $2^{-\Delta \Delta C q}$, was used to calculate fold amplification.
Enzyme-linked immune sorbent assay (ELISA). Cells were plated in 6-well plates (300,000 cells/well) and treated with $10 \mu \mathrm{g} / \mathrm{ml} \mathrm{hLL}-37$ in serum-free medium. After $48 \mathrm{~h}$, conditioned medium was collected for the SDF- $1 \alpha$ assay. The SDF- $1 \alpha$ assay was performed as described by the ELISA kit instructions (Raybiotech, Inc., Norcross, GA, USA) according to the manufacturer's recommendations.

Fluorescence-activated cell sorting (FACS). In order to assess CXCR4 expression, cells were subjected to surface and intracellular staining with a FITC-conjugated mouse anti-CXCR4 antibody (R\&D Systems, Inc., Minneapolis, MN, USA). Briefly, for surface staining, cells were harvested in trypsin/EDTA and washed in PBS. The cell suspension was then incubated with a CXCR4 antibody. For intracellular staining, cells were fixed with $2 \%$ paraformaldehyde for $20 \mathrm{~min}$ and then permeabilized with $0.1 \%$ saponin and $0.1 \%$ sodium azide in PBS for $20 \mathrm{~min}$. All staining was carried out on ice for $30 \mathrm{~min}$. The labeled cells were measured with a FACS Calibur (BD Biosciences, Franklin Lakes, NJ, USA) and analyzed using Win MDI software (Win MDI version 2.8).

Immunostaining. Cells were seeded on 4-well Lab-Tek II chamber slides from Nalgene Nunc International (Rochester, USA). After $12 \mathrm{~h}$, the chambers were replaced with conditioned media generated after $48 \mathrm{~h}$ from ASCs. After $18 \mathrm{~h}$, cells were fixed with $4 \%$ paraformaldehyde in PBS for 10 mins and then permeabilized for 5 mins with $0.1 \%$ Triton X-100 in PBS. Cells were then blocked with $1 \% \mathrm{BSA}$ in $\mathrm{PBS}$ at $37^{\circ} \mathrm{C}$ for 30 mins. Next, the cells were incubated with anti CXCR4 (sigma) for $30 \mathrm{~min}$ at $37^{\circ} \mathrm{C}$ in the dark, and then washed twice with PBS. This step was followed by incubation with an Alexa Fluor ${ }^{\circledR}$ 488 conjugated antibody (Invitrogen) for $30 \mathrm{~min}$ in the dark. A nucleic acid dye (DAPI; $0.5 \mu \mathrm{g} / \mathrm{ml}$ ) was then added to each slide for $5 \mathrm{~min}$ to stain the nuclei followed by washing with PBS. Finally, cells were viewed using an LSM700 confocal microscope system (Carl Zeiss Inc., Thornwood, NY, USA USA, x400 objective; ZEN lite software).

Statistical analysis. Statistical significance was estimated using the Student's t-test. $\mathrm{P}<0.05$ was considered to indicate a statistically significant difference. Results are shown as the mean \pm SD.

\section{Results}

Conditioned medium from LL-37 treated ASCs enhances HDF migration activity in vitro. Release of the LL-37 peptide in a wound is a physiologic response to cutaneous injury. To study the effects of CM from LL-37 treated ASCs on HDF migration, the migratory response of HDFs was assessed quantitatively by transwell migration assay. The migration of fibroblasts was markedly increased in CM from LL-37 treated ASCs, followed in order by CM from untreated ASCs and then control media. CM from LL-37 treated ASCs was found to induce HDF migration in a dose-dependent manner (Fig. 1A). The optimal dose of LL-37 for stimulating ASCs to induce migration in vitro was found to be $10 \mu \mathrm{g} / \mathrm{ml}$. In addition, we performed a time course evaluation of CM from LL-37 treated ASCs-induced cell migration at 12, 18, 24 and $36 \mathrm{~h}$. As shown 
in Fig. 1B, the level of HDF migration in the presence of $\mathrm{CM}$ from LL-37 treated ASCs increased in a time-dependent manner, with a maximum difference in migration observed at $24 \mathrm{~h}$. Together, these results suggested that CM from LL-37 treated ASCs can enhance HDF migratory response in a doseand time-dependent manner.

Conditioned medium from LL-37 treated ASCs enhances secretion of chemokine SDF-1 $\alpha$. Because SDF-1 $\alpha / C X C R 4$ has been shown the recruitment of HDFs involved in wound healing process, we examined whether SDF-1 $\alpha$, which is secreted by ASCs, plays a role in CM from LL-37 treated ASCs-induced HDF migration. As expected, the mRNA levels of SDF-1 $\alpha$ were significantly increased by CM from ASCs treatment with LL-37 in a dose-dependent manner (Fig. 2A). In addition, expression of SDF-1 $\alpha$ protein was markedly increased in CM from LL-37 treated ASCs in a time-dependent manner, with maximum expression noted $48 \mathrm{~h}$ after stimulation with $10 \mu \mathrm{g} / \mathrm{ml}$ of LL-37 (Fig. 2B).

Chemokine SDF-1a blockade decreases HDF migration activity. To characterize the involvement of SDF-1 $\alpha$ in CM from LL-37 treated ASCs-enhanced cell migration, we treated CM from LL-37 treated ASCs with a monoclonal anti-SDF- $1 \alpha$ neutralizing antibody. Blocking of SDF-1 $\alpha$ in this manner effectively decreased LL-37-induced SDF-1 $\alpha$ secretion from ASCs (Fig. 3A). Furthermore, photomicrographs revealed that CM from LL-37 treated ASCs induced HDF migration, while treatment with neutralizing SDF-1 $\alpha$ antibody followed by LL-37 stimulation resulted in significant inhibition of HDF migration (Fig. 3B and C).

Conditioned medium from LL-37 treated ASCs increases the expression of CXCR4 in HDFs. Because CXCR4 is a $\mathrm{CXC}$ chemokine receptor that is known to the recruitment of HDFs, we next examined the expression of CXCR4 in HDFs. Fig. 4A shows that CM from untreated ASCs produced a notable increase in the expression of CXCR4 compared to media control. Furthermore, expression of CXCR4 was further markedly increased by CM from LL-37 treated ASCs. The transcription of CXCR4 mRNA on HDFs reached a maximum at $6 \mathrm{~h}$ with CM from LL-37 treated ASCs. To investigate the effect of LL-37-inhibition in ASCs on CXCR4 expression in HDFs, CM from LL-37 treated ASCs were treated with a specific $\alpha$ LL-37 antibody, and the changes in CXCR4 expression were monitored by Real-Time PCR. As expected, the enhanced expression of CXCR4 observed in CM from LL-37-treated ASCs was significantly reduced in the presence of a neutralizing antibody ( $\alpha$ LL-37) (Fig. 4B). To further confirm the requirement of LL-37 in enhanced CXCR4 expression, ASCs were treated with pertussis toxin $(P t x), a G_{\alpha i}$ inhibitor, before activation with LL-37 to prevent SDF-1/CXCR4 signaling. Ptx treatment had a similar effect on CXCR4 expression in HDFs as the LL-37 antibody. Ptx treatment followed by LL-37 stimulation resulted in a significant inhibition of CXCR4 expression (Fig. 4B). In addition, we used flow cytometry to confirm that CM from LL-37 treated ASCs augments CXCR4 expression in HDF donor pools compared with CM from ASCs alone at $18 \mathrm{~h}$ (Fig. 5A and B). Taken together, our results indicated that CM from LL-37 treated
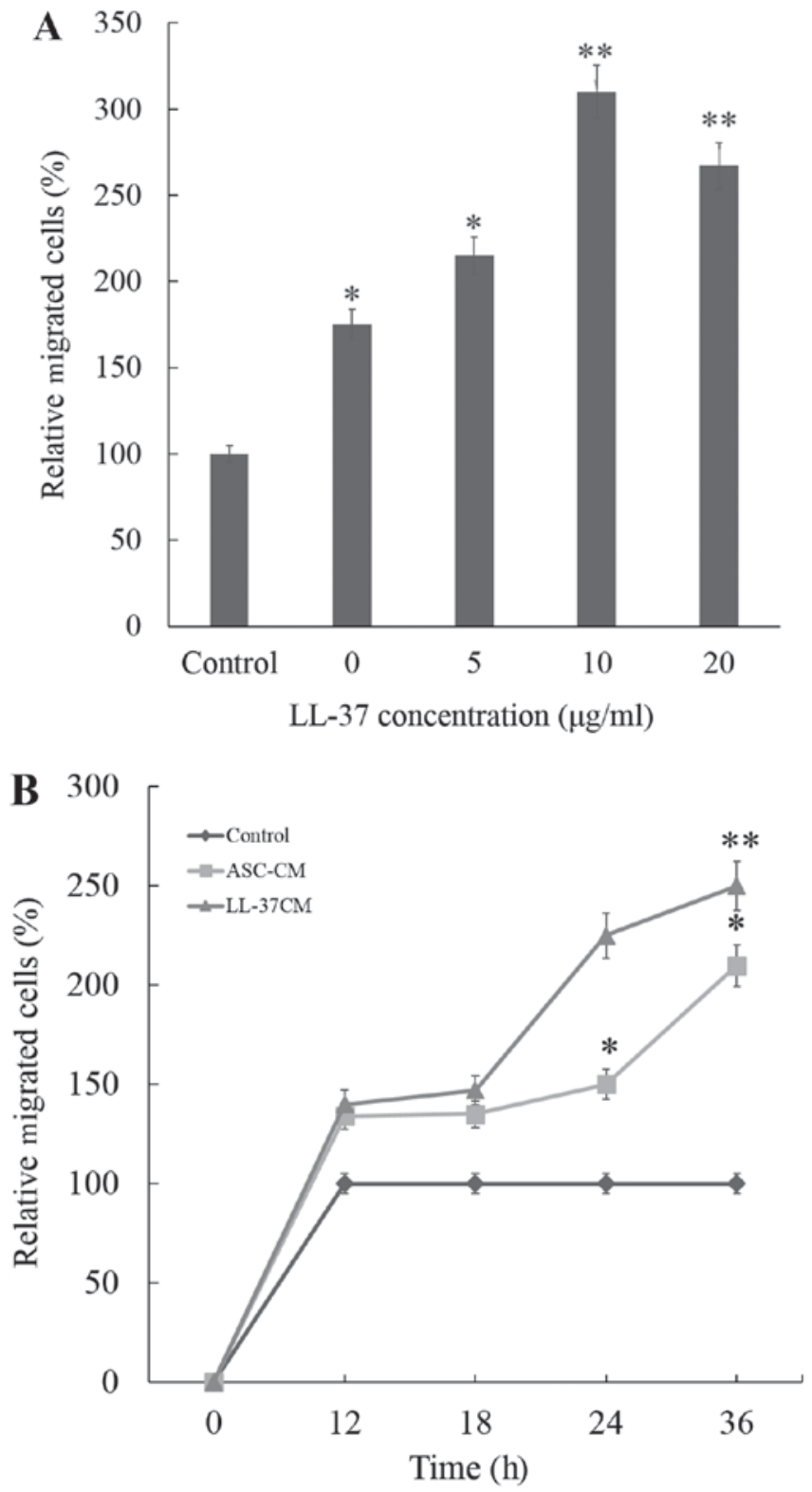

Figure 1. Effects of LL-37 on HDF migration in ASC-CM. (A) Dose titration of CM from LL-37-treated ASCs on induction of HDF migration as measured by transwell assay. HDFs were incubated with $0,5,10,20 \mathrm{ug} / \mathrm{ml}$ of ASC-CM or CM from LL-37-treated ASCs for $24 \mathrm{~h}$. The migratory effects of ASC-CM activated by $10 \mathrm{ug} / \mathrm{ml}$ LL-37 on HDFs were significantly higher compared with other concentrations. (B) HDF cells were incubated with media control (DMEM), ASC-CM, and CM from LL-37-treated ASCs in upper chamber of transwell inserts for $36 \mathrm{~h}$. Conditioned medium from LL-37-treated ASCs significantly enhanced migration of HDFs in a time-dependent manner, with maximum migration observed $24 \mathrm{~h}$ after stimulation. Statistical significance was estimated using the Student's t-test. Error bars in all figures represent the mean \pm SD from three independent experiments ( $\mathrm{P}<0.05$ vs. untreated cells, ${ }^{* * *} \mathrm{P}<0.05$ vs. ASC-CM)

ASCs enhanced the expression of CXCR4, which participates in HDF migration, at the mRNA and protein levels. These results suggest that $\mathrm{CM}$ from LL-37-treated ASCs may be a novel trophic factor for stimulating human HDF migration.

\section{Discussion}

Adipose stem cell-conditioned media (ASC-CM) contains a number of active peptides and has been successfully used to 

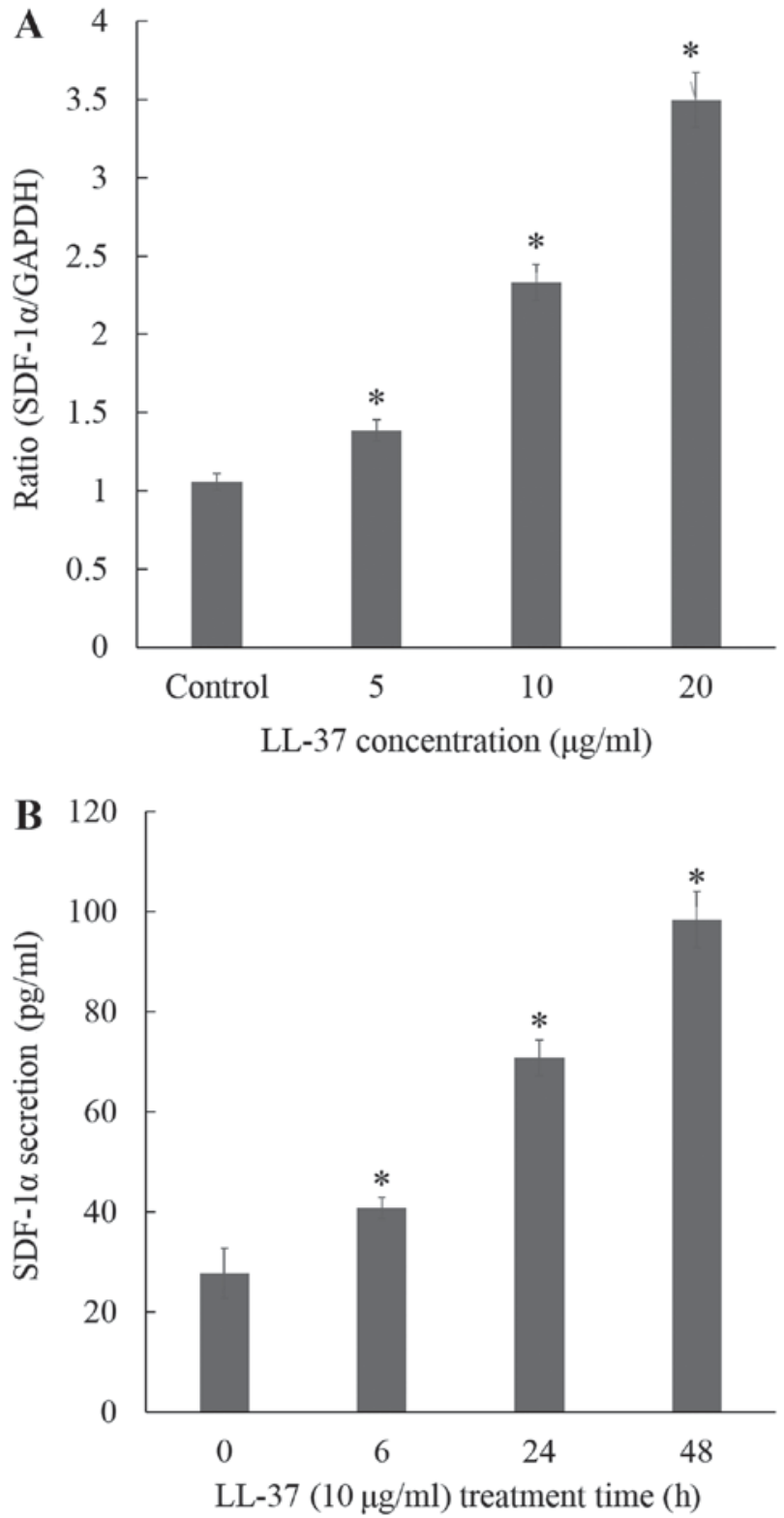

Figure 2. Effects of LL-37 on mRNA and protein levels of SDF-1 $\alpha$ in ASCs. (A) Administration of CM from LL-37-treated ASCs resulted in a dose-dependent SDF-1 $\alpha$ expression at mRNA. (B) SDF-1 $\alpha$ protein secretion by LL-37 increased in a time-dependent manner, with maximum expression observed $48 \mathrm{~h}$ after treatment with $10 \mathrm{ug} / \mathrm{ml}$ LL-37. Columns represent the mean percentage of SDF-1 $\alpha$ expression relative to the negative control. Statistical significance was analyzed using the Student's t-test. Error bars in all figures represent the mean \pm SD from three independent experiments ( $\mathrm{P}<0.05$ vs. untreated cells).

treat multiple types of tissue defects, including skin wounds both in vivo and in vitro $(1,3,6,10,11)$. A paracrine mechanism might be the most effective way for ASCs to promote wound healing (9). We hypothesized that ASC stimulated with LL-37 would increase expression of soluble factors promoting human dermal fibroblast migration, which is a key step in the wound healing process. Therefore, we set out to determine how ASCs would react to an LL-37-rich microenvironment to address whether the chemokine axis, which is well known to control cell migration properties, is influenced by CM from LL-37-treated ASCs, and whether it can regulate the migration of HDFs.
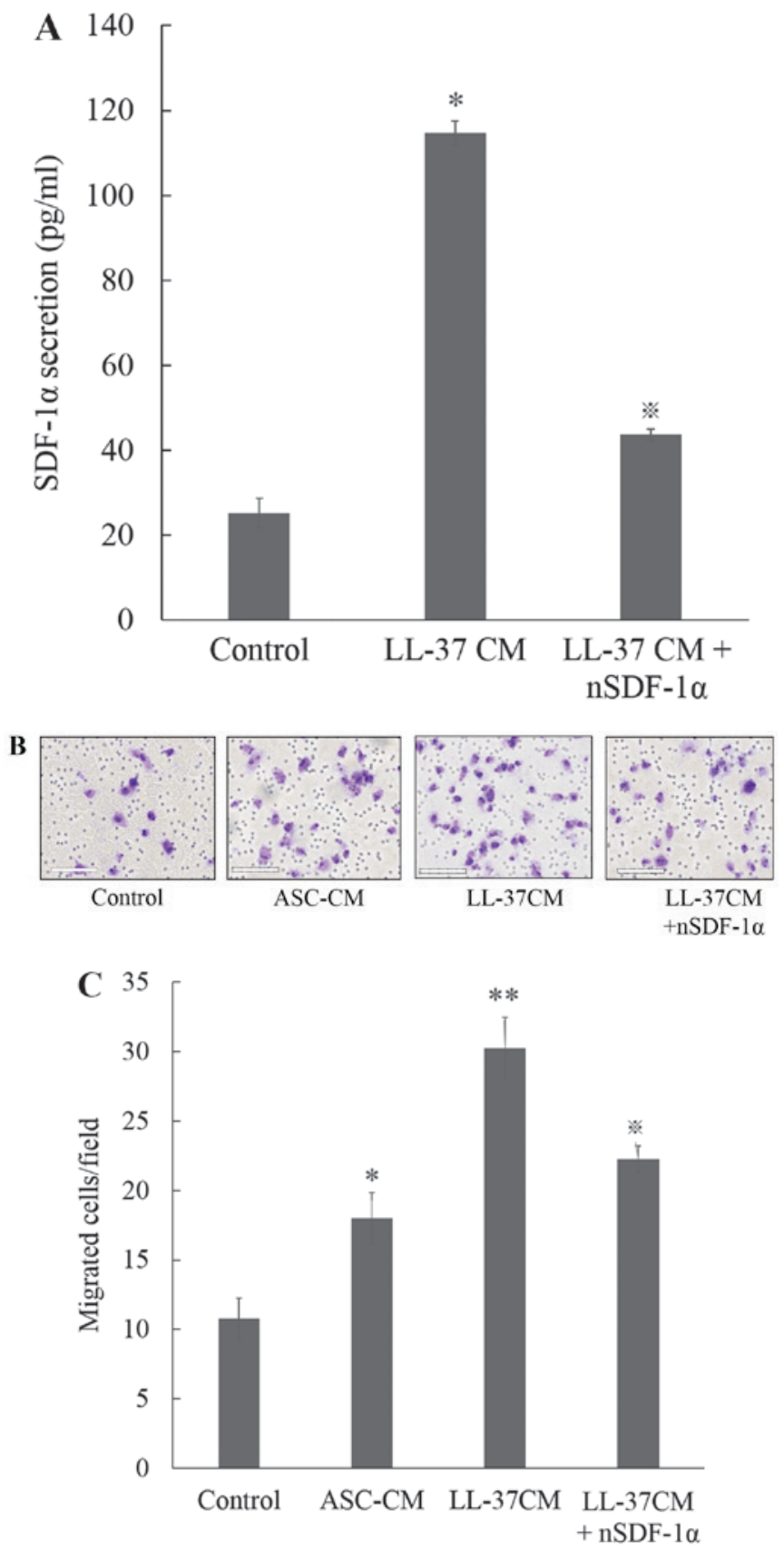

Figure 3. Effects of SDF-1 $\alpha$ blockade on HDF migration. (A) Enhanced expression of SDF-1 $\alpha$ was significantly reduced by a specific neutralizing SDF-1 $\alpha$ antibody. Columns represent the SDF-1 $\alpha$ secretion. (B) Representative images showing HDFs migrating toward control media, ASC-CM, CM from LL-37 treated ASCs, SDF-1 $\alpha$ neutralizing antibody (nSDF-1 $\alpha$ Ab)-treated CM from LL-37-stmulated ASCs. HDF cells were pretreated with CM from LL-37-treated ASCs and then treated with the nSDF-1 $\alpha$ antibody. (C) The relative percentages of migrating cells are indicated. The SDF-1 $\alpha$ neutralizing antibody effectively decreased the HDF migration inducing activity of CM from LL-37-treated ASCs. Columns represent the mean percentage of migrating cells relative to the negative control. Error bars in all panels represent the SD from three independent experiments. Statistical significance was estimated using the Student's t-test ( $\mathrm{P}<0.05$ vs. untreated cells, ${ }^{* *} \mathrm{P}<0.05$ vs. ASC-CM, ${ }^{*} \mathrm{P}<0.05$ vs. CM from LL-37-treated ASCs).

LL-37 is involved in wound healing from the first encounter with microbes to recovery of the tissue damaged during infection (12). LL-37 is stored at high concentrations $(40 \mu \mathrm{M}$ or $630 \mu \mathrm{g} / 10^{9}$ cells), predominantly in human neutrophil granules, and is up regulated in response to infections. The 
concentrations of LL-37 vary within different tissues and cells, and the physiological significance of the different activities of LL-37 has been actively debated. Ultimately, the functions of LL-37 in vivo are dependent on the peptide concentration and tissue composition at specific sites. The concentration-dependent effects of LL-37 range from anti-biofilm activity and an ability to block neutrophil apoptosis at low nM levels to antimicrobial and chemotactic effects at 0.1-10 $\mu \mathrm{M}$ levels, and cytotoxic effects at levels above $10 \mu \mathrm{M}$. Thus, defining the optimal efficacious concentration of a peptide within the wound environment is a challenge to the development of antimicrobial peptides for the treatment of wounds $(9,13)$. The optimal concentration of LL-37 that we found to stimulate ASC-CM to induce HDFs was $(10 \mu \mathrm{g} / \mathrm{ml})$ can be estimated as $2.2 \mu \mathrm{M}$. According to reported activities and the effects of LL-37 at particular concentrations, the concentration of LL-37 in this study may be considered not enough to develop chemotactic effects in vitro but sufficient to achieve chemotactic effects in vitro. Further investigation will be required to define the clinically effective doses of LL-37 needed to achieve functional augmentation of ASC-CM for treatment of wounds.

Phamacological preconditioning of stem cells to boost the release of cytoactive factors may represent the effective way to enhance their functional efficacy (14). Our experiments indicated that the paracrine effect of ASCs was significantly augmented by stimulation of media with LL-37. Regarding the results, we found that LL-37 stimulated SDF-1 $\alpha$ m RNA and secretion from ASCs, which play an important role in the HDFs migration since the neutralization of SDF-1 $\alpha$ block the stimulatory effects of CM from LL-37 treated ASCs. Evidence from our experiment supports the existence of secreting protein from ASC treated with LL-37 and the participant to regulate migration of HDFs. Identification of both active proteins from ASC-CM and their mechanism, as well as drug development using these peptides, will contribute to the establishment of effective ASC-CM therapeutic strategies for skin repair (4). Furthermore, a number of recent data suggest that the regulatory proteins involved in the regulation of self-renewal, growth, survival, and differentiation of premalignant neoplastic stem cells. Targeting of cancer stem cell using drugs that kill of permanently suppress these cells may be a prerequisite for the development of new curative treatment. Therefore, current research is seeking novel markers and targets that are preferentially expressed on cancer stem cells $(15,16)$.

We previously showed that CXC chemokine receptor 4 is essential for migration of HDFs (17). As expected, HDFs migration and expression of CXCR4/SDF-1 $\alpha$ on HDFs was significantly increased by CM from LL-37-treated ASCs followed by CM from untreated ASCs and control media, respectively. CXCR4 expression was enhanced by $\mathrm{CM}$ and the neutralizing experiments and Ptx treated data demonstrated that this effects were mediated by LL-37-G coupled protein receptor axis. However, we also found that cell migration was not decreased to the level of control media in the presence of neutralizing SDF-1 $\alpha$ (Fig. 3C). This observation contrasts with the dose-dependent relationship between LL-37 concentration and the secretion of active peptides such as SDF-1 $\alpha$ by human ASCs, suggesting that in addition to SDF-1 $\alpha$, other soluble factors and mechanisms may mediate the effects of LL-37 with respect to stimulation of HDF migration. For example, LL-37
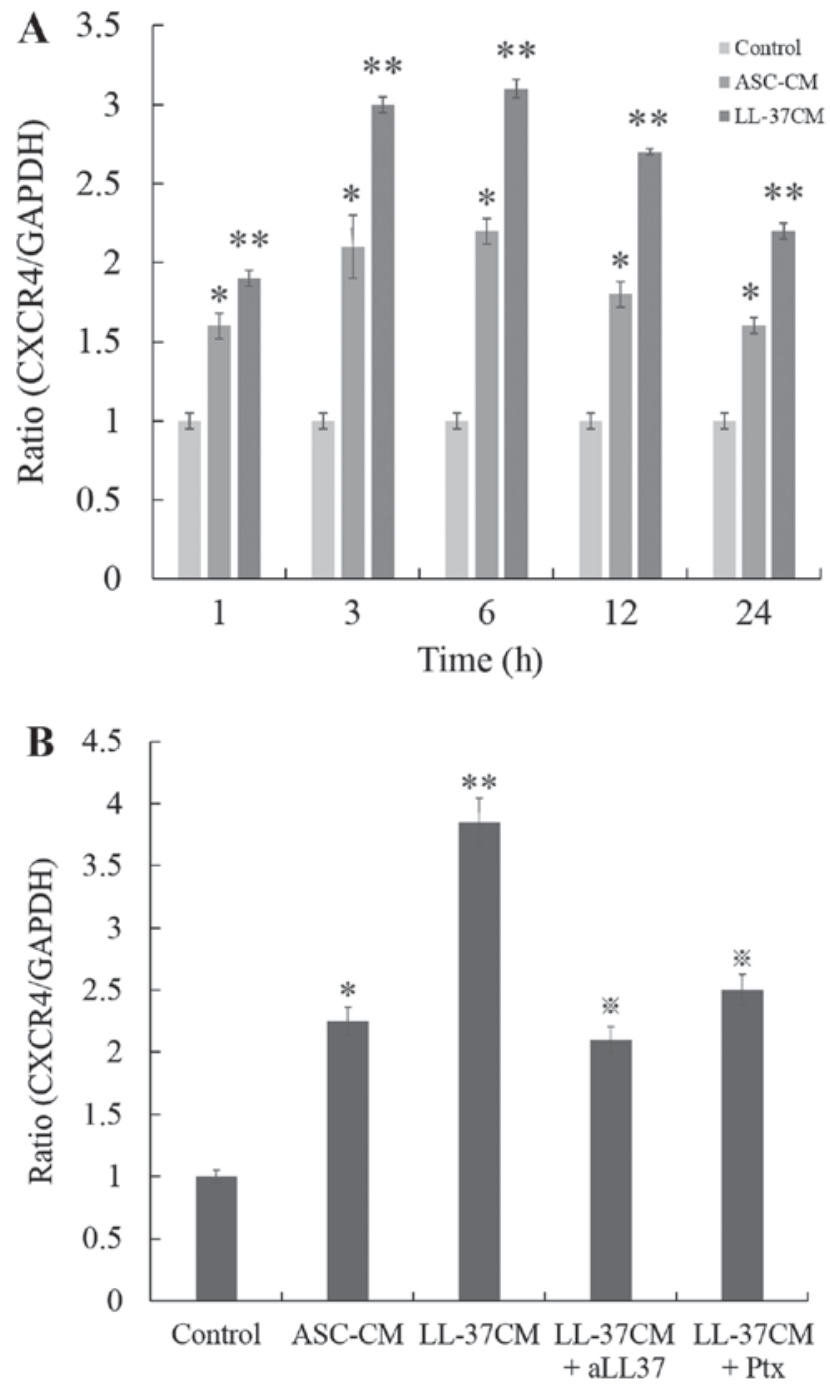

Figure 4. Effects of LL-37 on CXCR4 mRNA expression. (A) Therapeutic time window for ASC-CM and CM from LL-37-treated ASCs treatment. Administration of CM from LL-37-treated ASCs significantly increased CXCR4 expression compared with untreated ASC-CM. (B) The enhanced expression of CXCR4 of CM from LL-37-treated ASCs was significantly inhibited by LL-37 blockade with a specific $\alpha$ LL-37 antibody and pertussis toxin (Ptx). Error bars in all figures represent the mean \pm SD from three independent experiments. Statistical significance was estimated using the Student's t-test $\left({ }^{*} \mathrm{P}<0.05\right.$ vs. untreated cells, ${ }^{* *} \mathrm{P}<0.05$ vs. ASC-CM, ${ }^{*} \mathrm{P}<0.05$ vs. CM from LL-37-treated ASCs).

also leads to a considerable increase in secretion of monocyte chemoattractant protein-1 (MCP-1) in human ASCs (3). Thus, MCP-1 may affect not only multiple behaviors of human ASCs, including proliferation and migration, but also HDF migration. There still remains the possibility to have additional unknown factors in CM from ASCs to regulate CXCR4 in HDFs. Further investigations will be necessary to clarify the additional potential mechanisms and factors involved in CM from LL-37-treated ASCs induced migration of HDFs.

CXCR4 is a G-protein coupled receptor belonging to the CXC family of chemokine receptors. CXCR4 expression is dynamically regulated by external cues like hypoxia and can be upregulated following in vitro priming with a mixture of cytokine, as shown to enhance migration in vitro toward an SDF-1 $\alpha$ gradient $(18,19)$. Interaction of CXCR4 with its ligand, SDF-1 $\alpha$ directs the movements of cells in hematopoietic stem 

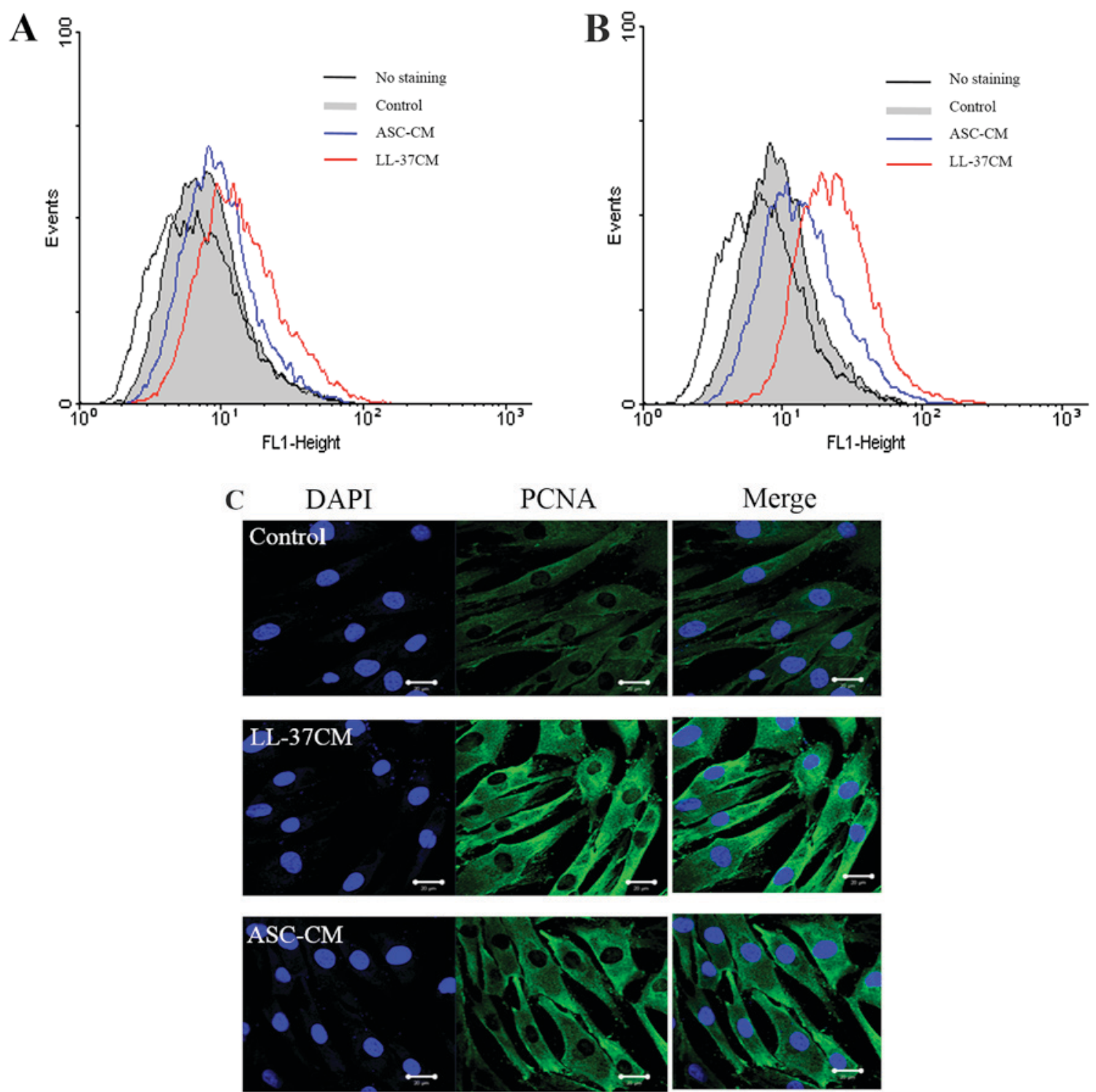

Figure 5. Effects of LL-37 on CXCR4 protein expression. (A) Surface expression of CXCR4 protein. Cells were treated with control, untreated ASC-CM, CM from LL-37-treated ASCs for $18 \mathrm{~h}$ and stained with an isotype control antibody for evaluation of CXCR4 surface expression. After incubation for $18 \mathrm{~h}$ surface staining was performed and analyzed by flow cytometry. The gray shaded area signifies the control, the blue line represents ASC-CM, and the red line represents CM from LL-37-treated ASCs. (B) Intracellular staining of CXCR4. Intracellular localization of CXCR4 protein in dermal fibroblast was considerably expressed by stimulated media (C) Immunostaining was performed and visualized using a confocal microscopy system, original magnification, $\mathrm{x} 400$.

cell homing, leukocyte trafficking, tumor metastasis (2,20-22). Manipulation of the CXCR4/SDF-1 $\alpha$ provides also clinical benefit to recipients of hematopoietic stem cell transplantation (23). It is postulated that CXCR4 axis control may also have a potential effect in HDF migration and skin repair. Recently, SDF-1 $\alpha$ exposure was shown to up regulate low basal CXCR4 surface expression in dermal fibroblast, which increased chemotaxis (17). Our results in the present study revealed that the expression of CXCR4 at mRNA levels was augmented by LL-37 stimulated ASC-CM followed by untreated ASC-CM and control media respectively, as shown to enhance migration toward an SDF-1 $\alpha$ gradient (Fig. 4). Nevertheless, present study showed discrepancy between mRNA and protein expression of CXCR4 in dermal fibroblast induced by LL-37 stimulated ASC-CM. Although CXCR4 mRNA showed the enhanced expression, flow cytometry data indicated no significant expression level of CXCR4 protein
(Fig. 5A). Instead of surface expression, intracellular localization of CXCR4 protein in dermal fibroblast was considerably expressed by stimulated media (Fig. 5B). The prominent intracellular localization of CXCR4 suggests that dynamic equilibrium between the cytoplasm and plasma membrane may modulate CXCR4 availability at the cell surface, and thus cell responsiveness to SDF-1 $\alpha$ gradient (24). Although CXCR4 undergoes internalization after interaction with ligand, like other G-protein coupled receptor, the extent of intracellular expression and endocytosis kinetics differs from one cell type to the other $(24,25)$.

In conclusion, this study demonstrated that conditioned medium from LL-37-treated ASCs accelerated migration of HDFs by upregulating CXCR4 expression in vitro. This is the first report to demonstrate the ameliorative effect of conditioned medium from LL-37-treated ASCs on HDFs migration. Blocking LL-37 with pertussis toxin and a neutralizing 
LL-37 antibody markedly reduced HDF cell migration. Together, these data suggested that conditioned medium from LL-37-treated ASCs enhances CXCR4 expression in HDFs, which may possibly stimulate cutaneous wound healing. These results also suggest that conditioned medium from LL-37-treated ASCs may be an effective wound healing therapeutic candidate. Our data also clarified that SDF-1 is one of the active soluble factors responsible for mediating the effects of conditioned medium from LL-37-treated ASCs in wound healing. In addition, the effect of mechanism of LL-37 treated ASC-CM on HDFs in mouse model will be investigated in future studies.

\section{References}

1. Kim WS, Park BS, Sung JH, Yang JM, Park SB, Kwak SJ and Park JS: Wound healing effect of adipose-derived stem cells: A critical role of secretory factors on human dermal fibroblasts. J Dermatol Sci 48: 15-24, 2007.

2. Qu Y, Mao M, Li X, Zhang L, Huang X, Yang C, Zhao F, Xiong Y and $\mathrm{Mu} \mathrm{D}$ : Enhanced migration and CXCR4 over-expression in fibroblasts with telomerase reconstitution. Mol Cell Biochem 313: 45-52, 2008

3. Chen L, Tredget EE, Wu PY and Wu Y: Paracrine factors of mesenchymal stem cells recruit macrophages and endothelial lineage cells and enhance wound healing. PLoS One 3: e1886, 2008.

4. Nie C, Yang D, Xu J, Si Z, Jin X and Zhang J: Locally administered adipose-derived stem cells accelerate wound healing through differentiation and vasculogenesis. Cell Transplant 20: 205-216, 2011.

5. Kim WS, Park BS and Sung JH: The wound-healing and antioxidant effects of adipose-derived stem cells. Expert Opin Biol Ther 9: 879-887, 2009.

6. Heilborn JD, Nilsson MF, Kratz G, Weber G, Sørensen O, Borregaard $N$ and Ståhle-Bäckdahl $M$ : The cathelicidin anti-microbial peptide LL-37 is involved in re-epithelialization of human skin wounds and is lacking in chronic ulcer epithelium. J Invest Dermatol 120: 379-389, 2003.

7. Coffelt SB, Marini FC, Watson K, Zwezdaryk KJ, Dembinski JL, LaMarca HL, Tomchuck SL, Honer zu Bentrup K, Danka ES Henkle SL and Scandurro AB: The pro-inflammatory peptide LL-37 promotes ovarian tumor progression through recruitment of multipotent mesenchymal stromal cells. Proc Natl Acad Sci USA 106: 3806-3811, 2009.

8. Murakami M, Ohtake T, Dorschner RA, Schittek B, Garbe C and Gallo RL: Cathelicidin anti-microbial peptide expression in sweat, an innate defense system for the skin. J Invest Dermatol 119: 1090-1095, 2002.

9. Duplantier AJ and van Hoek ML: The human cathelicidin antimicrobial peptide LL-37 as a potential treatment for polymicrobial infected wounds. Front Immunol 4: 143, 2013.

10. Hu L, Zhao J, Liu J, Gong N and Chen L: Effects of adipose stem cell-conditioned medium on the migration of vascular endothelial cells, fibroblasts and keratinocytes. Exp Ther Med 5: 701-706, 2013
11. Cho JW, Kang MC and Lee KS: TGF- $\beta 1$-treated ADSCs-CM promotes expression of type I collagen and MMP-1, migration of human skin fibroblasts, and wound healing in vitro and in vivo. Int J Mol Med 26: 901-906, 2010.

12. Vandamme D, Landuyt B, Luyten W and Schoofs L: A comprehensive summary of LL-37, the factotum human cathelicidin peptide. Cell Immunol 280: 22-35, 2012.

13. Nijnik A and Hancock RE: The roles of cathelicidin LL-37 in immune defences and novel clinical applications. Curr Opin Hematol 16: 41-47, 2009.

14. Liu GS, Peshavariya HM, Higuchi M, Chan EC, Dusting GJ and Jiang F: Pharmacological priming of adipose-derived stem cells for paracrine VEGF production with deferoxamine. J Tissue Eng Regen Med 10: E167-E176, 2016.

15. Schulenburg A, Blatt K, Cerny-Reiterer S, Sadovnik I, Herrmann H, Marian B, Grunt TW, Zielinski CC and Valent P: Cancer stem cells in basic science and in translational oncology: Can we translate into clinical application? J Hematol Oncol 8: 16, 2015.

16. Wang X, Wang C, Zhang X, Hua R, Gan L, Huang M, Zhao L, $\mathrm{Ni} \mathrm{S}$ and Guo W: Bmi-1 regulates stem cell-like properties of gastric cancer cells via modulating miRNAs. J Hematol Oncol 9: 90, 2016.

17. Yang Y, Shim SK, Kim HA, Seon M, Yang E, Cho D and Bang SI: CXC chemokine receptor 4 is essential for Lipo-PGE1-enhanced migration of human dermal fibroblasts. Exp Dermatol 21: 75-77, 2012.

18. Schioppa T, Uranchimeg B, Saccani A, Biswas SK, Doni A, Rapisarda A, Bernasconi S, Saccani S, Nebuloni M, Vago L, et al: Regulation of the chemokine receptor CXCR4 by hypoxia. J Exp Med 198: 1391-1402, 2003

19. Kucia M, Jankowski K, Reca R, Wysoczynski M, Bandura L, Allendorf DJ, Zhang J, Ratajczak J and Ratajczak MZ: CXCR4-SDF-1 signalling, locomotion, chemotaxis and adhesion. J Mol Histol 35: 233-245, 2004.

20. Herzig DS, Driver BR, Fang G, Toliver-Kinsky TE, Shute EN and Sherwood ER: Regulation of lymphocyte trafficking by CXC chemokine receptor 3 during septic shock. Am J Respir Crit Care Med 185: 291-300, 2012

21. Suratt BT, Petty JM, Young SK, Malcolm KC, Lieber JG, Nick JA, Gonzalo JA, Henson PM and Worthen GS: Role of the CXCR4/SDF-1 chemokine axis in circulating neutrophil homeostasis. Blood 104: 565-571, 2004.

22. Koczulla R, von Degenfeld G, Kupatt C, Krötz F, Zahler S, Gloe T, Issbrücker K, Unterberger P, Zaiou M, Lebherz C, et al: An angiogenic role for the human peptide antibiotic LL-37/hCAP-18. J Clin Invest 111: 1665-1672, 2003.

23. Green MM, Chao N, Chhabra S, Corbet K, Gasparetto C, Horwitz A, Li Z, Venkata JK, Long G, Mims A, et al: Plerixafor (a CXCR4 antagonist) following myeloablative allogeneic hematopoietic stem cell transplantation enhances hematopoietic recovery. J Hematol Oncol 9: 71, 2016.

24. Pelekanos RA, Ting MJ, Sardesai VS, Ryan JM, Lim YC, Chan JK and Fisk NM: Intracellular trafficking and endocytosis of CXCR4 in fetal mesenchymal stem/stromal cells. BMC Cell Biol 15: 15, 2014

25. Ding Z, Issekutz TB, Downey GP and Waddell TK: L-selectin stimulation enhances functional expression of surface CXCR4 in lymphocytes: Implications for cellular activation during adhesion and migration. Blood 101: 4245-4252, 2003 